\title{
El Programa de Mary Richmond y las bases fundamentales de la metodología profesional
}

\author{
Mary Richmond's Program and the fundamental bases of professional \\ methodology \\ Enrique DI CARLO ${ }^{1}$ \\ Universidad Nacional de Mar del Plata
}

Recibido: $19 / 05 / 2011$

Revisado: $19 / 05 / 2011$

Aceptado: 05/06/2011

Disponible on line: 29/09/2011

\section{Resumen}

En este trabajo se presenta un análisis y una síntesis de ciertas ideas de la obra de Mary Richmond, quien merece junto a G. Hamilton, H. Perlman o Ch. Towle una consideración fundamental como fundadora de la profesión. Su obra concentra, a nuestro entender, una gran sabiduría metodológica, filosófica y social. Se hará referencia al esfuerzo de la autora por otorgar un status científico a la profesión y desarrollar las bases de su metodología, a los valores que inspiran el Trabajo Social, su concepción de sujeto Richmond y el análisis de dos aspectos totalmente integrados: su inspiración igualitaria y junto con esta la defensa de la individuación.

Palabras clave: Richmond, metodología, valores, individualización, igualdad.

\begin{abstract}
This article presents an analysis and synthesis of certain ideas from the work of Mary Richmond who deserves, along with G. Hamilton, H. Perlman or Ch. Towle, fundamental respect as a founder of the profession. To our understanding, her work brings to bear a great methodological, philosophical and social wisdom. The article refers to the author's efforts to accord a scientific status to the profession and to develop the bases of its methodology, to the values that inspire Social Work, its conception of the Richmond subject and analyses of two completely integrated aspects: her egalitarian inspiration along with her defense of individuation.
\end{abstract}

Keywords: Richmond, methodology, values, individuation, equality.

Referencia normalizada: Di Carlo, E. (2011). «El Programa de Mary Richmond y las bases fundamentales de la metodología profesional». Cuadernos de Trabajo Social, 24: 47-55.

Sumario: Introducción. 1. El programa de Mary Richmond. 2. Referencias bibliográficas.

\section{Introducción}

En este capítulo, vamos a sintetizar ciertas ideas de algunos autores que podemos considerar clásicos y fundadores de la profesión. Son autores del Trabajo Social Profesional (TSP) que merecen nuestra consideración fundamental. La obra de cada uno de ellos concentra, a nuestro entender, una gran sabi- duría metodológica, filosófica y social. En general, han sido utilizados tradicionalmente en la formación de profesionales de Trabajo Social, también en Argentina, y contienen un importante conjunto de conocimientos que consideramos necesario actualizar en este momento. Se trata de: Mary Richmond, Gordon Hamilton, Charlotte Towle y Helen Perlman².

${ }^{1}$ El prof. Di Carlo falleció el 3 de septiembre de 2008.

2 Para los fines del tema central del presente monográfico, sólo se seleccionaron las páginas que hacen referencia a Mary Richmond. 
Las autoras desarrollan sus pensamientos principalmente en relación con el caso social individual. Hemos encontrado en ellas las bases fundamentales de la metodología profesional, que hemos retomado en parte, para desarrollarla en nuestras investigaciones sobre el método de trabajo con grupos.

Muy explícitamente nuestro trabajo de investigación, durante diez años consecutivos con un grupo profesional en la Universidad de la República de Uruguay, estuvo orientado a desarrollar la investigación del Trabajo Social con grupos desde las perspectivas de la metodología profesional, como ya expresamos, presentes de manera más general en los escritos de Trabajo Social individual ${ }^{3}$.

Hasta ese momento, la enseñanza académica del método de Trabajo Social con grupos se parecía más, en casi todos los casos en nuestros medios, a una Psicología social o a un curso sobre dinámicas de grupo. Es un trabajo que sentimos que debía hacerse para evitar la sustitución de la metodología específica profesional por otra cualquiera. En Argentina, por ejemplo, los cursos de Trabajo Social con grupos eran, en general, exposiciones sobre el enfoque operativo de Pichón Rivière, enfoque que consideramos interesante, pero muy diferente del profesional de Trabajo Social. Expresamente el enfoque operativo de Pichón Rivière está destinado a tratar la incidencia de las operaciones y resistencias en el aprendizaje y no al Trabajo Social. La escasa bibliografía universal disponible sobre el nivel grupal en Trabajo Social (Trecker, Konopka, Wilson y Rylan, etc.), contribuía a este fenómeno.

Intentamos seguir el mismo esquema en nuestras investigaciones oficiales, desde el año 1993, relativas al trabajo con comunidades en la Universidad Nacional de Mar del Plata (Argentina). Con otro equipo profesional hemos tratado de profundizar en el proceso de Trabajo Social con comunidades barriales con cierto éxito. También en este caso, el trabajo con comunidades, desde el punto de vista profesional, estaba ocupado en general por enfoques sociológicos, dentro de los cuales la profesión jugaba un papel relativamente accesorio. Este enfoque sociológico, que empezó a expandirse a partir del desarrollismo como corriente política internacional, no tuvo mucho éxito en nuestro país como transformación de las comunidades reales ni para el desarrollo de las mismas. Dentro de él la metodología del Trabajo Social quedó como algo anexo y subsumido.

Este artículo -se nos sabrá disculpar por la amplitud de las citas de los autores tratados- se debe a los argumentos ya indicados y además a una voluntad de propiciar la lectura o relectura de los mismos, que pueden tener funciones reconciliadoras con el Trabajo Social profesional, pese a tantos prejuicios actualmente existentes en nuestros medios. Asimismo, sentimos la necesidad de comunicar que la relectura y estudios de los textos clásicos citados nos volvieron a asombrar por la profundidad de sus análisis y por lo que dejan de saldo positivo para la labor científica cotidiana de los trabajadores sociales. Son especialmente recomendables y constituyen además un buen instrumento para la docencia con los nuevos estudiantes del Trabajo Social profesional.

\section{El Programa de Mary Richmond}

Tal vez sea interesante introducir algunos datos biográficos de esta autora central para el Trabajo Social. Nos basaremos en algunas -pocas- fuentes, a saber: los archivos de la Russell Sage Foundation de Nueva York, la National Asociation Social Worker de Estados Unidos (NASW) y en el prólogo de Natalio Kisnerman al libro de la autora Caso Social Individual, publicado en español en 1993.

Mary Richmond nació en Belleville, Illinois, EE.UU., en el año 1861. Después de sus estudios secundarios en Baltimore ingresó como tesorera, en 1889, en la institución dedicada a la filantropía, la Charity Organization Society (COS). Dos años más tarde pasó a ser

3 Véanse los numerosos artículos y los siguientes libros de Di Carlo, E. (1969). Perspectivas en la Conducción de Grupos. Montevideo: Guillaumet. Del mismo autor (1971). Una Investigación sobre la Orientación de Grupos. Montevideo: Guillaumet. Así como (1971). Necesidades Básicas y Cambio Social. Montevideo: Guillaumet; (1976). El Trabajo Social: Teoría - Metodología - Investigación. Buenos Aires: ECRO. 
Secretaria General de la misma, incorporándose al equipo de las «visitadoras amigables» y a tales efectos, concurrió a las conferencias para capacitarse. Tomó conciencia de las dificultades para elevar el nivel de conocimiento de las «visitadoras» y comenzó entonces estudios de Sociología y Filosofía. Le influyó especialmente el pensamiento de William James y de John Dewey. Conoció y trabó una sólida amistad con George Mead, circunstancia que influiría en la obra de ambos. Tomó contacto con la obra de Freud y con la de los antropólogos culturalistas del momento.

Como Secretaria General de la filial de la COS se propuso el objetivo de transformarla, trabajando con ahínco en ese cargo durante diez años en diversos temas para la creación de legislaciones en torno a problemas de la familia. Insistió en la creación de una Escuela para trabajadores sociales. La COS concretó esa idea en 1898 con la creación de la Escuela de Filantropía de Nueva York a la que Mary Richmond se incorporó como docente. En 1907 volvió a Nueva York, vinculándose en calidad de investigadora a la Russell Sage Foundation y asumió la dirección del Departamento de Caridad de dicha fundación, integrándose también en el Comité de Instrucción de la Escuela de Filantropía.

Dictó numerosas conferencias en su país y propició el crecimiento institucional de la profesión en distintos aspectos hasta que, en 1917, publicó el libro Social Diagnosis, obra pilar inicial de la profesión del Trabajo Social profesional. El inicio para el Trabajo Social que constituye esta obra es al mismo tiempo la conclusión de diecisiete años de investigación e intervención de campo de Mary Richmond. Por fin, en 1918, la Escuela de Filantropía pasó a llamarse Escuela de Trabajo Social, dirigiendo Mary Richmond la cátedra de Caso Social Individual. Esta autora en 1921 recibió el Master of Arts del Smith College en reconocimiento de su tarea.

Estos datos biográficos pueden ampliarse en las fuentes citadas y fundamentalmente en el libro reciente de Bibiana Travi, La dimensión técnico-instrumental en trabajo social. (2006), libro recomendable por su riqueza en muchos aspectos.

Como todos sabemos, se le adjudica a Mary Richmond el papel de haber sido la principal fundadora del Trabajo Social profesional moderno. No significa que nuestra vuelta a ella se deba a un interés puramente histórico, sino a que esta autora, como casi todos los clásicos de cualquier disciplina, configura un punto de partida alto, con tantas condiciones de capacidad, de dominio de perspectivas sobre el asunto tratado, unidas generalmente a expresiones inspiradoras de aprecio por el camino que se propone recorrer. Capacidad e inspiración son características que encontramos en la autora y que, al releerla, todavía nos asombra por su despejada lucidez en puntos que preadvirtió desde el primer momento, a principios del siglo XX. Asuntos tales como, por ejemplo, la preocupación expresa por los riesgos que correría el Trabajo Social profesional al pasar de las asociaciones sociales de entonces a la orbita estatal: el temor a una creciente burocratización y politización del mismo: el riesgo de que se pudieran poner al servicio de los intereses de los partidos políticos dominantes.

Sabemos ahora que tal peligro no era imaginario, por más que nos resulte en un balance general muy positivo el paso del Trabajo Social profesional a la órbita estatal.

Para que los trabajadores sociales especializados en el servicio de casos individuales entren de buena gana y en buen número en el servicio de los poderes públicos, es necesario que estos den alguna seguridad de continuidad en los métodos seguidos y cierta garantía de independencia entre los partidos políticos. Una vez cumplida esta condición puede ser que (...) cuantas formas del servicio social de casos individuales haga progresos más considerables. Servirán seguramente a una clientela mas numerosa que bajo nuestra dirección privada (Richmond, 1993, p. 166).

A continuación veremos la importancia de los alcances y de los que pueden llamarse valores permanentes en el principio de la profesión protagonizado por Mary Richmond. Nos basaremos fundamentalmente en dos obras: Social Diagnosis (1917), y What is Social Case Work? (1922). El análisis de estas obras nos permite comprender su arduo esfuerzo creador de teoría y práctica para la profesión; como sucede con casi todos los clásicos, su trabajo incita a la reverencia respetuosa. Sin embargo, sabemos que no es este tratamiento el que se 
dispensa en general y muchos menos en Latinoamérica, donde muchas veces el desprecio por los orígenes históricos de Trabajo Social profesional corre a la par con la ignorancia sobre el tema.

El primer punto que se destaca es su intención y tarea concreta de formar una profesión científica con un personal capacitado, frente a una realidad caracterizada por un conjunto disperso, generalmente inefectivo y muchas veces hasta mórbido, de la llamada caridad como había funcionado hasta ese momento.

Este programa, que contó con algunos antecedentes precientíficos en cierto sentido positivos y que se apoyó en determinadas filosofías provenientes de la Ilustración ${ }^{4}$, constituyó un esfuerzo creativo revolucionario para la época $\mathrm{y}$, en algún sentido, lo sigue siendo hoy en día.

Mary Richmond tuvo que cuestionar la teoría del hombre dominante en su época, para suplantarla por una concepción del ser humano como sujeto potencialmente responsable de su destino, en condiciones de encuadre generalmente dificultosas o adversas. Concibe al hombre como un ser libre. Es algo constante en toda su obra el principio de no constituirse en la providencia del otro y el de propiciar, en cambio, su crecimiento racional como persona. Este principio encaja justamente con el principio pedagógico moderno que comienza a fundarse en el modernismo con «Emilio o sobre la educación» de Jean Jacques Rousseau y que impregnará posteriormente toda la educación de tendencia igualitaria, especialmente la educación social de la que es representante el Trabajo Social profesional. En este sentido se recomienda la lectura del trabajo de Bibiana Travi, Primeras aproximaciones para la comprensión de la naturaleza, fundamentos y formas del Trabajo Social en la obra de Mary Richmond (Di Carlo et al., 2005).

No existe ninguna razón grave que nos impida desempeñar el papel de una providencia particular frente a un animal, con la condición de que nos demos cuenta de sus debilidades y de que no lo abandonemos en medio del camino cuando nos cansemos de ser buenos. Pero todo nos prohíbe actuar del mismo modo con un ser humano. Se encuentra el motivo si se examina la manera como funciona la inteligencia del hombre. El lactante parece equipado de forma menos adecuada para sus necesidades que un gatito, un perrito o cualquier otro animalito y su desarrollo mental es mucho más lento. La forma relativamente automática con que el animal responde a los estímulos externos, sigue caminos bien delineados, es lo que llámanos reacciones instintivas. Significa que en el animal, el progreso mental está confinado en un círculo que limita al joven ser y lo hace incapaz de adquirir necesidades progresivas y elevadas. El hombre no está encerrado en este círculo y es reemplazado en él por una espiral. Sus reacciones son mucho más lentas porque ya en el comienzo de su carrera, está obligado por una necesidad imperiosa a comparar un concepto con otro y a deducir un tercero; en otros términos: debe razonar. Las operaciones del conocimiento y de la formación de las costumbres le hacen salir del círculo estrecho de las reacciones instintivas para que siga una espiral que se amplía sin cesar con nuevas complicaciones que agrandan su horizonte y le otorgan la facultad de comunicarse con lo visible e invisible a la vez. La diferencia entre el círculo y la espiral es la diferencia que existe entre la rutina y la acción deliberada, entre el animal doméstico y el pionero en busca de descubrimientos (...) Interponerse entre un hombre y el estímulo que lo empuja a emprender un acción reflexiva, es perjudicarlo mucho más gravemente que cuando hablamos del peligro de pauperizarlo (...). Hace tiempo que tengo la idea de que no existe en el servicio social una especialidad que tenga como objeto a la donación como ayuda (Richmond, 1993, pp. 110-115).

En el prólogo de lo que será su explicación metodológico-profesional, Richmond nos muestra explícitamente, con un ejemplo excepcional, una parte sustantiva del método. A Hellen Keller la señorita Sullivan no la trató como a un ser empobrecido e inferior susceptible de ayuda a raíz de su ceguera, ni sustituyó su propia humanidad por ayudas paralizantes, como eran las tendencias propias del sentido común sentimental. Lo que hizo Sullivan fue apelar a las capacidades potenciales de Keller para su desarrollo como persona. Algo obviamente más difícil de hacer y de lograr que de predicar.

${ }^{4}$ Véase el capítulo: Valores de base constitutivos del TSP. En E. Di Carlo et al. (2005) La Profesión del Trabajo Social, Tomo I. 
Todavía hoy podemos observar conductas frente a la persona en situación de discapacidad que oscilan entre la represión y el sentimentalismo frustrante. Gran parte de la educación familiar está amenazada todavía por unos errores como éstos que cuesta mucho erradicar en el mundo. Hay que reconocer que también observamos todavía a algunos trabajadores sociales mal formados que se consideran una autoridad legítima para dictaminar sobre la vida del asistido, contraviniendo entonces a uno de los principios rectores del programa transformador de Mary Richmond en la educación social: el del respeto a la personalidad del otro. Este respeto no consiste en una postura pasiva frente a los problemas del otro, una posición contemplativa, sino que se desenvuelve como solidaridad activa y comprometida con el otro (sea un individuo, una familia, un grupo o un sector social).

No se trata de un respeto místico a no se sabe qué parte de la personalidad del otro, sino de un respeto a su condición de ser pensante, que es lo que definitivamente le hace ser humano con capacidad de discernir entre una posibilidad y otra, entre un camino u otro. Capacidad de discernir y de autoorientarse que atañe a las condiciones prudenciales de la existencia, cuando se toma consciencia, por ejemplo, de «que la mejor manera de defender la vida es el trabajo y que la mejor manera de criar los hijos es con amor»; y también tiene alcance moral en todo lo concerniente a elegir formas de vida no agresivas, sino respetuosas y cooperadoras. La capacidad racional crítica de discernir entre posibilidades diferentes y procesos diversos abre siempre disyuntivas muy especiales en sus significaciones para nuestro bienestar y para nuestra moralidad. Esta característica universal, este sentido común del hombre, «su racionalidad», es reconocida como el principio de su humanidad y encuentra formulaciones supremas en la filosofía kantiana, que influirá en Mary Richmond a través de George Mead entre otros.

Es decir, el programa de Mary Richmond, como todo el Trabajo Social tradicional, es el de capacitar al hombre en sus prácticas de toma de decisiones, tanto para defender su vida como para realizarse como agente moral. Esta unidad entre la preocupación por lo vital y la humanidad del sujeto no es una carac- terística de lujo para algunas conciencias privilegiadas, sino la condición de lo humano mismo que se desvela como responsabilidad educativa.

Si bien no todos los hombres reciben condiciones de desarrollo adecuadas: los más pobres generalmente carecen de ellas y los ricos suprimen su capacidad de discernimiento muchas veces y lo sustituyen por un esnobismo desorientado. A los primeros se les niega la posibilidad de discernir y este hecho, en el medio subdesarrollado, sucede de forma alarmante por las carencias de trabajo, nutrición, educación e ideales de vida. Son los destinatarios principales de la tarea profesional del Trabajo Social, aunque la destrucción de la clase superior, la banalidad, el consumismo desenfrenado, etc., no pueden quedar completamente fuera de la esfera de preocupación de los profesionales y, menos aún, en cuanto influyen sobre las otras clases sociales (Guillebaud, 2001).

Mary Richmond abre un nuevo camino profesional, el del Trabajo Social como una profesión de cambio del otro y de sus circunstancias, basándose en la hipótesis de una racionalidad potencial a la que apelar en todo sujeto. Es su teoría del hombre y del método. Ahora bien, la autora comprende desde el primer momento la relación entre los cambios individuales y los cambios de la sociedad como una totalidad. Esta idea ordena casi todo su pensamiento y estará presente en el Servicio Social tradicional en casi todos los autores:

Aunque el trabajador social goza de un cierto grado de reconocimiento gracias al desempeño de una actividad útil para la comunidad, se ve limitado por el hecho de que su público desconoce la diferencia existente entre pretender hacer algo y hacerlo realmente. «Hacer el bien era la vieja expresión que definía el servicio social. Se trata de una petición de principio, al igual que las expresiones mas recientes de «servicio social» y de «trabajo social» -a menos que la sociedad sea realmente servida. Debemos congratularnos, pues, de que los trabajadores sociales ya no basan sus demandas de respeto únicamente en las buenas intenciones. Para poder realizar un análisis critico de su tarea, hemos de evaluar sus grandes esfuerzos desde la distancia y debemos instarles a valorar su trabajo mediante los mejores criterios proporcionados por la experiencia- criterio que a pesar de sus imperfecciones actuales, están pro- 
gresando hacia un punto que nos permitirá calificarlos de profesionales.

Los trabajadores sociales de los Estados Unidos forman un gran grupo ocupacional. La mayor parte desempeña su actividad en la esfera del trabajo social de caso -es decir, una actividad cuyo objetivo inmediato es la mejora de la situación de individuos o familias, uno a uno, y no la mejora del colectivo. No obstante, mejora colectiva y mejora individual son interdependientes; la reforma social y el trabajo social de caso progresan necesariamente juntos. Esta verdad fundamental se reiterara en varias ocasiones a medida que el presente estudio sobre diagnostico social avance (Richmond, 2005, pp. 2-3)

\section{En este mismo sentido, la autora señala:}

No creo que se pueda dar un mejor consejo a la asistente del servicio familiar de casos individuales que el de estudiar y desarrollar la parte de su esfera de la actividad que atañe a las investigaciones sociales o a la mejora «en conjunto de las masas». No quiero decir que ellas deban renunciar a su propia tarea ni descuidarla, a fin de emprender estudios especiales o lanzarse en campañas legislativas, pero entiendo con esto que su actividad debe ser mas fecunda desde el punto de vista científico de lo que es actualmente; que los descubrimientos sociales deben ser el producto accesorio de su servicio provechoso en el terreno de casos individuales, para emplear una frase de Sheffield y que debería constituirse en testigos fieles de la necesidad de reformas sociales cada vez que esta necesidad se manifiesta en el curso de su trabajo diario. Deberían también proporcionar argumentos que preparan al público para la reforma.

La exploración social debe ya al servicio social de casos individuales diversas reformas relativas a las viviendas y a las primeras campañas emprendidas contra la tuberculosis. El servicio social familiar ha contribuido, también como lo indicaremos mas adelante, a la legislación del trabajo con los niños (...) agreguemos «en fin» las investigaciones ya publicadas sobre el abandono conyugal y sobre la negativa de sostener a la familia. No hay duda de que los trabajadores sociales especializados en el servicio familiar se encuentran colocados para hacer observaciones de primera intención, si no están 1 sobrecargados de trabajo, y que su formación teórica en el terreno de las ciencias sociales, unida a su formación práctica en el campo del servicio social, les permite situar bien sus estudios (Richmond, 2005, pp. 150-151).

En el programa de Mary Richmond que estamos analizando se destacan claramente dos aspectos. Por un lado su inspiración igualitaria y junto con ésta la defensa de la individuación. El punto de vista igualitario se expresa de manera muy explícita en sus adhesiones al marco histórico progresista de actuación para cuyo análisis nos remitimos de nuevo al escrito de Bibiana Travi (2006), y a los escritos de Mary Richmond:

Es fácil sentirse satisfecho de los resultados del servicio social, si nos conformamos con los primeros síntomas de mejoramiento o si juzgamos estos resultados desde un solo punto de vista, pero no si nos atrevemos a examinar como punto de vista la vida en conjunto, pensando constantemente en el bienestar permanente del individuo y de la sociedad (Richmond, 1993, p. 62).

En estas páginas, hemos hecho menor hincapié en el gran número de reformas sociales que han sido fruto directo del Trabajo Social y en muchas otras que le deben su eficaz modificación o su buena gestión (Richmond, 2005, p. 427).

Aunque la autora utiliza varias veces el concepto de masa para referirse a lo general humano en la sociedad, toda su filosofía va en contra de lo que más tarde se entenderá como masa, el hombre masificado que tanta críticas ha recibido en la época contemporánea. Por el contrario, tanto Mary Richmond como el Trabajo Social posterior defienden acérrimamente el principio de individuación concerniente a la persona como fundamento de su ser y de la convivencia democrática, algo completamente en polémica con el concepto de masa:

El éxito, en la empresa especial conocida bajo el nombre del servicio social de casos individuales, exige un alto grado de la facultad de percepción de la esencia que le es propia a cada ser humano. Entre los dones naturales de todo trabajador social especializado en el servicio de casos individuales, debe figurar una deferencia instintiva para la personalidad y sobre todo para las personalidades que le son mas 
extrañas. Establecer un modelo de excelencia y exigir con que se conformen con el mismo, no es su finalidad. Es, por el contrario, su privilegio descubrir y liberar lo mejor que existe en cada individuo, amar profundamente el dibujo infinitamente variado de la humanidad y esforzarse con alma de artista, en desarrollar la profundidad y la riqueza de todo lo que lo conforman (1993, p.106).

Vemos entonces cómo el Trabajo Social Profesional hace desde el inicio una gran defensa de la individuación y con ella de la autodeterminación. Con esto se diferencia de toda igualación en clases o categorías de hombres y se acerca a la crítica del hombre masificado.

Esta crítica que -como dijimos-es propia de la conciencia contemporánea, encontró tal vez su máxima expresión en el Movimiento del 68 francés, que con la máxima de que «lo único que está prohibido es prohibir», con la revolución de la imaginación, puso en marcha un amplísimo movimiento mundial. Este fenómeno por su complejidad no puede ser ampliamente comentado en este artículo, aunque queremos realizar algunas reflexiones sobre todo lo concerniente al Trabajo Social. En un sentido podría considerarse contradictoria la idea de individuación-autodeterminación con la de adaptación, que encontramos en gran parte de los escritos iniciales de Trabajo Social y que figura como un elemento básico de la definición del Trabajo Social profesional en el texto de Mary Richmond.

Mientras los seres humanos sigan siendo humanos y su medio siga siendo el mundo no se podrá imaginar un estado de cosas en el que ellos mismos y el medio en que ellos viven dejen de necesitar adaptaciones y readaptaciones particulares, condensados en una fórmula de pensamiento que precede. Llego a esta tentativa de definición: el servicio social de casos individuales es el conjunto de métodos que desarrolla la personalidad, reajustando consciente e individualmente al hombre a su medio social (p. 67).

Sólo nos cabe consignar que la fórmula «lo único que esta prohibido es prohibir», como forma de vida en la que todo es posible, parece ser una expresión más bien de una clase alta intelectualizada que de ser humano común. El hombre común sabe que con su integridad y libertad necesita estar comunicado y aceptar a la realidad en muchos más sentidos. La idea de adaptación que promueven originariamente los asistentes sociales tiene que ver con un comportamiento social según el cual el sujeto acepta participar en normas respetuosas para con sus semejantes (muchas veces abandonando la agresividad de la delincuencia que no tiene nada de original) integrándose así en la comunidad.

A nuestro entender, entonces, aunque la idea de individuación como diferencia es central para el sujeto y para la convivencia democrática, puede llegar a justificar muchas locuras sociales y fracasos existenciales, sobre todo relacionales ${ }^{5}$.

Si nadie con sinceridad quiere tener, por ejemplo un hijo inadaptado en la escuela, en casa, con sus conocidos, compañeros, maestros, etc., no hay que tener miedo a usar el concepto de adaptación. En realidad lo que se opone a la masificación es la humanización,

5 Diario Clarín. República Argentina. 26 de Julio de 2006. Artículo de Marcelo A. Moreno: «¿Es civilizado legalizar al degenerado?»: «Por lo menos desde los años 60, ciertos países del norte de Europa suelen ser tomados como modelos de sociedades avanzadas. Y en verdad Suecia, Noruega, Finlandia, Dinamarca y Holanda hicieron suficientes méritos como para ser consideradas así. Esas naciones construyeron sociedades que se transformaron en ejemplos de solidez institucional, justicia social, ecuánime distribución de la riqueza y respeto por los derechos humanos. Es decir, genuinos paradigmas de civilización en un mundo surcado por el salvajismo y caos. Pero quizás el rasgo más distintivo de estas democracias sociales es la tolerancia. Allí las minorías fueron y son respetadas a raja tabla y las libertades individuales, inviolables. Pero el 18 de Julio último Holanda pasó un límite que quizá incluya todos los límites. Según informa el diario inglés The Guardian ese día un tribunal autorizó la formación de un partido político formado por pedófilos, esto es, individuos cuyo objeto de deseo sexual son los niños. Así la formación «Amor Fraterno, Libertad y Diversidad» podrá participar en las elecciones de noviembre, con el sólo requisito de presentar candidatos y 500 firmas de adherentes. Su programa nos es complicado: pugna por bajar la edad de consentimiento legal para las relaciones sexuales hasta los 12 años, quiere legalizar la pornografía infantil y permitir que ésta se emita por TV.» 
que contiene la tolerancia y el respeto a las diferencias, y lo que se opone al individualismo extremo es la participación en el ser en común, algo que podemos reconocer como necesidad y disfrutar. Uno no pierde ni enajena su individualidad, por participar en un coro vocal, solamente se integra a «un nosotros» ampliado. Esta es la concepción de la relación entre universalidad e individuación humana que orienta el Trabajo Social desde el principio, que va desde la afirmación de una convivencia pluralista (democrática), hasta el respeto de búsquedas exclusivas de la individualidad.

Veamos estas ideas: igualdad, individuación y autodeterminación y cómo se expresa en el verbo de Mary Richmond:

Los trabajadores sociales deben mirar de frente este hecho capital: que existen diferencias individuales que no se pueden desarraigar entre los hombres. La democracia también debe tener en cuenta este hecho y la enseñanza no debe jamás perderlo de vista. Afirmar que diferimos los unos de los otros parece una verdad baladí, ya que no solamente debemos contar con las diferencias heredadas e invariables, sino también con todas las disimilitudes provenientes de la diversidad de nuestras impresiones en el curso de nuestra vida social (...) Pocas verdades evidentes son tan a menudo descuidadas por los hombres del estado, por los administradores públicos, por la masa y, lamento decirlo, por los mismos trabajadores sociales. Es otro hecho, correlativo al primero, que sigue llamando nuestra atención: la existencia de una naturaleza común a todos los hombres. Tenemos por otra parte, razón de recordar esta verdad en el caso de que solo la acción y el tratamiento en masa puedan llevarnos a resultados que nos proponemos (p. 100).

Como ya expresamos, estas ideas proceden del marco intelectual de la Ilustración, momento histórico en el que la relación de respeto al semejante pasa de la esfera de lo religiosamente recomendado a la interiorización de lo socialmente exigible, tal y como aparece en la Declaración de los Derechos del Hombre y del Ciudadano (1789):

Los representantes del pueblo francés, constituidos en Asamblea nacional, considerando que la ignorancia, el olvido o el menosprecio de los derechos del hombre son las úni- cas causas de las calamidades públicas y de la corrupción de los gobiernos, han resuelto exponer, en una declaración solemne, los derechos naturales, inalienables y sagrados del hombre, a fin de que esta declaración, constantemente presente para todos los miembros del cuerpo social, les recuerde sin cesar sus derechos y sus deberes; a fin de que los actos del poder legislativo y del poder ejecutivo, al poder cotejarse a cada instante con la finalidad de toda institución política, sean mas respetados y para que las reclamaciones de los ciudadanos, en adelante fundadas en principios simples e indiscutibles, redunden siempre en beneficio del mantenimiento de la Constitución y de la felicidad de todos.

En consecuencia, la Asamblea nacional reconoce y declara, en presencia del Ser Supremo y bajo sus auspicios, los siguientes derechos del hombre y del ciudadano:

Artículo primero: Los hombres nacen y permanecen libres e iguales en derechos. Las distinciones sociales sólo pueden fundarse en la utilidad común.

Artículo segundo: La finalidad de toda asociación política es la conservación de los derechos naturales e imprescriptibles del hombre. Tales derechos son la libertad, la propiedad, la seguridad y la resistencia a la opresión (...).

Del mismo modo, tal y como planteamos en La profesión de Trabajo Social (2005):

Es en este marco del pensar moderno y contemporáneo desde donde surge el Trabajo Social Profesional. La primera declaración aprobada de los Derechos del Hombre y el Ciudadano en el siglo XVIII romano, consagra al hombre como el fundamento de la nueva sociedad. Son los derechos del hombre y el ciudadano, entre los que se destacan la libertad de acción, pensamiento y expresión.

Sencillamente: ¿Puede considerarse que alguien que se crea superior a otro pueda ser un trabajador social? ¿Que alguien que manipule a otro pueda ser un trabajador social? ¿Que alguien que fomente la dependencia y sumisión pueda ser trabajador social? ¿Que alguien intolerante con creencias religiosas diversas pueda ser un trabajador social? Y así podríamos seguir definiendo al trabajador social por todos lo valores de la conciencia moderna y contemporánea. (Di Carlo, 2005, pp. 154-156). 


\section{Referencias bibliográficas}

Di Carlo, E. et al. (2005). La profesión de Trabajo Social. Mar del Plata: PAIDEIA-Universidad Nacional de Mar del Plata.

Guillebaud, J. C. (2002). El principio de la humanidad. Madrid: Espasa-Calpe.

Richmond, M. (1917). Social Diagnosis. Londres: The Free Press - Nueva York: Collier-Macmillan.

Richmond, M. (1993). Caso Social Individual. Buenos Aires: Humanitas, (1 ${ }^{\text {a }}$ ed. 1922, Russell Sage Foundation, Nueva York).

Richmond, M. (2005). Diagnóstico Social. Madrid: Siglo XXI Editores (1ª ed. 1917, Nueva York: Russell Sage Foundation).

Travi, B. (2006). La dimensión técnico-instrumental en Trabajo Social. Reflexiones y propuestas acerca de la entrevista, la observación, el registro y el informe social. Buenos Aires: Espacio.

Travi, B. (2005). Primeras aproximaciones para la comprensión de la naturaleza, fundamentos y formas del Trabajo Social en la obra de Mary Ellen Richmond. En E. Di Carlo.et al. La profesión de Trabajo Social. Mar del Plata: PAIDEIA-Universidad Nacional de Mar del Plata. 